\title{
CBX7, a Potential Prognostic Biomarker in Lung Adenocarcinoma
}

\author{
Yanlong Yang ${ }^{1} * *$ \\ Zaoxiu $\mathrm{Hu}^{2}$,* \\ Hongwen Sun' \\ Qinghe $\mathrm{Yu}^{\prime}$ \\ Linzhu Yang' \\ Fang Yin' \\ Yongmen Sun' \\ Lisha $\mathrm{Pu}^{3}$ \\ Xingming Zhu' \\ Shen $\mathrm{Li}^{4}$ \\ Xiaobo Chen' \\ Yunping Zhao' \\ 'Department of Thoracic Surgery, The \\ First Affiliated Hospital of Kunming \\ Medical University, Kunming, 650032, \\ People's Republic of China; ${ }^{2}$ Department \\ of Pathology, The Third Affiliated \\ Hospital of Kunming Medical University \\ (Yunnan Cancer Hospital, Yunnan Cancer \\ Center), Kunming, 650II8, People's \\ Republic of China; ${ }^{3}$ Department of \\ Nephrology, Kunming Yanan Hospital, \\ Kunming, 65005I, People's Republic of \\ China; ${ }^{4}$ Department of Thoracic Surgery, \\ Baoshan People's Hospital, Baoshan, \\ 678000, People's Republic of China
}

*These authors contributed equally to this work
Correspondence: Xiaobo Chen; Yunping Zhao

Department of Thoracic Surgery, The First Affiliated Hospital of Kunming Medical University, No. 295 Xichang Road, Wuhua District, Kunming, 650032, Yunnan Province, People's Republic of China $\mathrm{Tel} / \mathrm{Fax}+8687165324888$

Email chenxb@ydyy.cn; zhaoypcq@।26. com
Background: Lung adenocarcinoma (LUAD) is a major type of NSCLC and has high morbidity and mortality. The identification of useful prognostic biomarkers for LUAD is important. $\mathrm{CBX} 7$ has been reported in various cancers yet its expression level and potential roles have not been fully understood.

Methods: GEPIA, Oncomine, TCGA, KM plotter and OSluca databases were used to explore the expression profile and prognostic effects of CBX7 mRNA expression in patients with LUAD. TIMER was used to explore the relationship between CBX7 and immune infiltrating cells. GSEA was used to further explore the potential biological process and pathways regulated by $\mathrm{CBX} 7$ in LUAD. Lastly, IHC detection of CBX7 in 95 samples was used to validate the result.

Results: We found CBX7 was downregulated in LUAD in GEPIA, Oncomine and TCGA databases. TCGA, KM plotter and OSluca databases suggested that CBX7 was associated with poor clinical outcomes and low survival rate. Using TIMER, we found that CBX7 might be associated with immune infiltration. Via gene set enrichment analysis, we found that tumor-associated biological processes and signaling pathways were enriched in the CBX7 downregulated group. Using clinical samples, we found that $\mathrm{CBX} 7$ protein has low expression in LUAD and was associated with poor survival.

Conclusion: $\mathrm{CBX} 7$ might serve as a promising biomarker and potential molecular target in LUAD.

Keywords: $\mathrm{CBX} 7, \mathrm{LUAD}$, prognosis, biomarker, immune infiltration

\section{Introduction}

Lung cancer has the highest morbidity and mortality not only in China but also worldwide. About $80 \%$ of lung cancers are non-small-cell lung cancers. ${ }^{1}$ NSCLC consisted of 3 subtypes, including lung adenocarcinoma (LUAD), lung squamous cell carcinoma (LUSC), and large cell carcinoma. ${ }^{2}$ Among these, LUAD has been widely accepted as the dominant subtype, especially among Asian women. ${ }^{3}$ Though various efforts have been made, the prognosis of lung cancer remains poor. In addition, clinical outcomes may vary even when similar treatments are applied to patients with the same histology and TNM stages. Such instability could be caused by genetic intra-tumor heterogeneity, which has been a major obstacle for lung cancer treatment. ${ }^{4}$ As considerable progress is being made regarding molecular biological techniques, such as next-generation sequencing, it is now possible to further understand the molecular features in lung cancer.

Polycomb group (PcG) complexes are epigenetic regulatory complexes that conduct transcriptional repression of target genes via chromatin modification. Polycomb 
Table I Comparison of CBX7 in LUAD and Normal Lung Tissues Across 5 Datasets in Oncomine

\begin{tabular}{|l|l|l|l|l|}
\hline Dataset & ADC/Normorl Lung & Fold Change & t-test & P value \\
\hline Hou et al,20I0 & $45 / 65$ & -2.539 & -12.164 & $1.54 \mathrm{E}-19$ \\
Su et al,2007 & $27 / 30$ & -2.786 & -8.358 & $3.36 \mathrm{E}-1 \mathrm{I}$ \\
Selamat et al,2012 & $58 / 58$ & -2.374 & -13.417 & $1.53 \mathrm{E}-22$ \\
Landi et al,2008 & $58 / 49$ & -2.344 & -13.103 & $7.50 \mathrm{E}-24$ \\
Okayama et al,2012 & $226 / 20$ & -1.749 & -9.735 & $9.93 \mathrm{E}-\mathrm{II}$ \\
\hline
\end{tabular}

repressive complexes 1 and 2 (PRC1 and PRC2) are the two major forms of $\mathrm{PcG}$, which are required for maintaining the stemness of embryonic stem cells and numerous types of adult stem cells. Abnormally increased expression of either PRC1 or PRC2 triggers aberrant silencing of corresponding target genes, which leads to the appearance of a broad spectrum of carcinomas. ${ }^{5,6}$ Chromobox7 (CBX7) belongs to the CBX protein family, which is involved in the organization of polycomb repressive complex 1 (PRC1). All of these proteins are involved in the regulation of heterochromatin, gene expression, and developmental progress. ${ }^{5}$ Recent studies have reported that $\mathrm{CBX} 7$ expression is decreased in many cancers, such as cervical cancer, ${ }^{7}$ pancreatic cancer, ${ }^{8}$ thyroid, colorectal, and breast cancer. ${ }^{5}$ However, the expression profile and prognostic value of CBX7 in LUAD samples remains unclear at present. This study aims to explore the differential expression and potential role of CBX7 in LUAD.

Therefore, in the current research, we investigated the expression profile and prognostic effects of $\mathrm{CBX} 7 \mathrm{mRNA}$
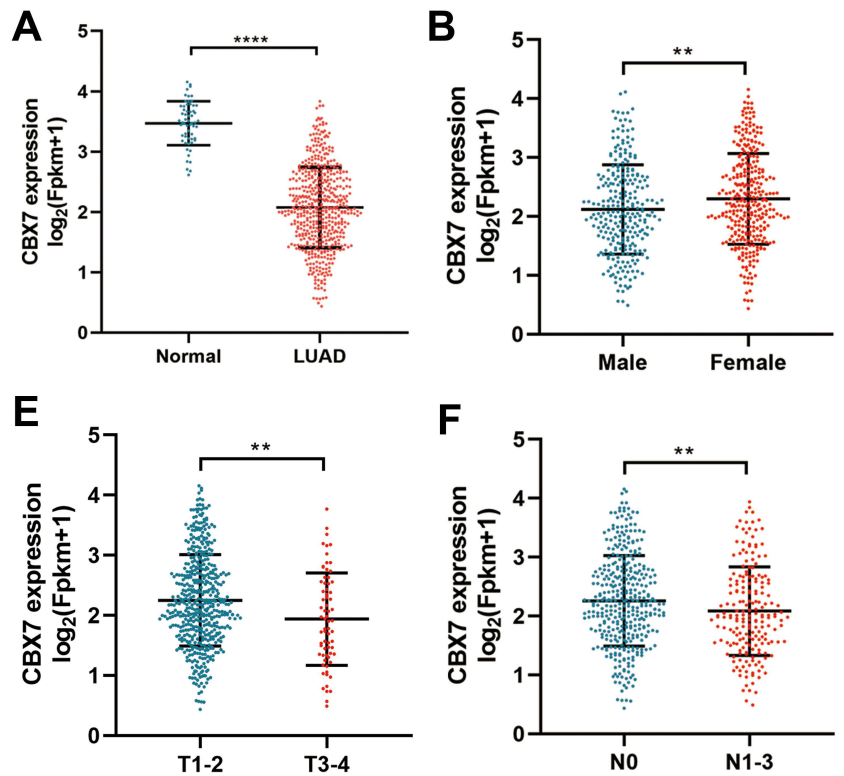

expression in patients with LUAD using various databases, including TCGA, Oncomine, GEPIA, OSluca and KaplanMeier plotter. TIMER was used to explore the relationship between $\mathrm{CBX} 7$ and immune infiltrating cells. GSEA was used to further explore the potential biological processes and pathways regulated by CBX7 in LUAD. Lastly, IHC detection of CBX7 in 95 samples was used to validate the result. Overall, our research preliminarily yet systematically characterizes the expression profiles of $\mathrm{CBX} 7$ in LUAD and reveals that the detection of the CBX7 expression status in LUAD patients is instructive and may be applied as potential biomarkers for prognostic assessment.

\section{Materials and Methods Data Mining in TCGA}

The UCSC Xena browser (https://xenabrowser.net/) was used to download the Cancer Genome Atlas (TCGA) dataset of lung adenocarcinoma (LUAD). The expression level of $\mathrm{CBX} 7$ mRNA expression
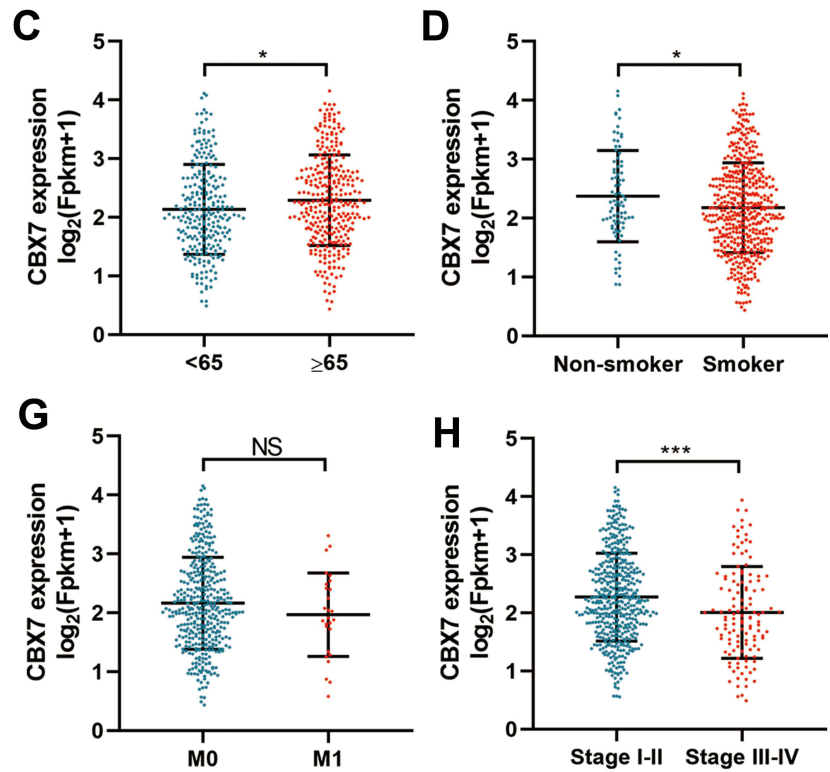

Figure I The expression level of CBX7 in LUAD and its relationship with clinicopathological characteristics in TCGA. (A). CBX7 was downregulated in LUAD when compared with normal tissues. (B-H) the relationship between CBX7 expression and sex (B), age (C), smoking status (D), T (E), N (F), M (G) and stage (H). *, ** and *** represented $\mathrm{P}<0.05,0.01$ and 0.001 respectively. NS represented not significance. 
Table 2 The Relationship Between CBX7 and the Clinicopathological Characteristics of Patients with LUAD in TCGA Dataset

\begin{tabular}{|c|c|c|c|}
\hline Parameters & Low $(N=246)$ & High ( $N=249)$ & $\mathbf{P}$ \\
\hline Age & $63.72 \pm 10.4$ & $66.8 \pm 9.5$ & 0.001 \\
\hline Sex & & & \\
\hline Female & $120(48.8 \%)$ & $156(58.4 \%)$ & 0.03 \\
\hline Male & $\mid 26(5 \mid .2 \%)$ & III(4I.6\%) & \\
\hline Smoking History & & & \\
\hline $2 / 3 / 4 / 5$ & $209(85.0 \%)$ & $215(80.5 \%)$ & 0.33 \\
\hline 1 & $30(12.2 \%)$ & $45(16.9 \%)$ & \\
\hline No data & $7(2.8 \%)$ & $7(2.7 \%)$ & \\
\hline $\mathrm{T}$ & & & \\
\hline TI-T2 & 206(83.7\%) & $240(89.9)$ & 0.047 \\
\hline T3-T4 & $39(15.9 \%)$ & $25(9.4 \%)$ & \\
\hline Tx & $\mathrm{I}(0.4 \%)$ & $2(0.7 \%)$ & \\
\hline $\mathrm{N}$ & & & \\
\hline No & $|4|(57.6 \%)$ & $189(70.8 \%)$ & $<0.001$ \\
\hline $\mathrm{NI}-3$ & $102(41.6 \%)$ & $69(25.8 \%)$ & \\
\hline $\mathrm{Nx}$ & $2(0.8 \%)$ & $9(3.4 \%)$ & \\
\hline$M$ & & & 0.688 \\
\hline Mo & $232(94.3 \%)$ & $253(95.5 \%)$ & \\
\hline MI & |4(5.7\%) & $12(4.5 \%)$ & \\
\hline Pathologic Stage & & & $<0.001$ \\
\hline I-II & I76(7|.5\%) & $220(82.4 \%)$ & \\
\hline III-IV & $69(28.0 \%)$ & $4 I(15.4 \%)$ & \\
\hline No data & $\mathrm{I}(0.4 \%)$ & $6(1.4 \%)$ & \\
\hline Recurrence status & & & 0.039 \\
\hline Living & $118(48.0 \%)$ & $158(59.2 \%)$ & \\
\hline Dead & $81(32.9 \%)$ & $69(25.8 \%)$ & \\
\hline No data & $47(19.1 \%)$ & $40(15.0 \%)$ & \\
\hline Living Status & & & 0.003 \\
\hline Living & $136(55.3 \%)$ & $183(68.5 \%)$ & \\
\hline Dead & $105(42.7 \%)$ & $76(28.5 \%)$ & \\
\hline No data & $5(2 \%)$ & $8(2.5 \%)$ & \\
\hline
\end{tabular}

level in tumor and normal lung tissues was collected. In addition, the clinicopathological characters and survival status were collected.

\section{Oncomine and GEPIA Database Analysis}

538 samples from 5 Oncomine datasets (www.oncomine. $\underline{\text { org }})^{9}$ were used to analyze the CBX7 expression in LUAD vs normal tissues. Gene Expression Profiling Interactive Analysis (GEPIA, http://gepia.cancer-pku.cn/) ${ }^{10}$ was used to explore the expression profile in pan-cancers.

\section{Meta-Analyses of CBX7 Expression in} GEO Dataset in OSluca Online Database

The OSluca online database (http://bioinfo.henu.edu.cn/ LUCA/LUCAList.jsp) $^{11}$ contains multiple lung cancer datasets, which can perform hazard ratio analysis on the impact of CBX7 in LUAD. The hazard ratio (HR) and its 95\% confidence interval (CI) in each eligible dataset were extracted for meta-analysis. The combined HRs were estimated graphically by Forest plots. Heterogeneity between studies was detected by the $\mathrm{Q}$ test and the $\mathrm{I}^{2}$ metric (no heterogeneity: $\mathrm{I}^{2}=0-25 \%$; moderate heterogeneity: $25-$ 50\%; large heterogeneity: 50-75\%; and extreme heterogeneity: $75-100 \%$ ). If $\mathrm{P} \geq 0.10$ in the Q test or $\mathrm{I}^{2}<50 \%$, the fixed-effect model (the Mantel Haenszel method) ${ }^{12}$ was used. Otherwise, a random effect model analysis was conducted. ${ }^{13}$ Subgroup analyses based on sex, age, smoking status and stage were conducted. In addition, publication bias was assessed by the method reported by Begg and Mazumdar. ${ }^{14}$ The meta-analysis was conducted by STATA v11.2 (StataCorp, College Station, Texas, USA).

\section{Data Mining in Kaplan-Meier Plotter}

Kaplan-Meier plotter (www.kmplot.com) ${ }^{15}$ was used to analyze the relationship between CBX7 mRNA expression and overall survival (OS) as well as recurrence-free survival (RFS). The hazard ratio (HR) with 95\% confidence intervals (CI) and log-rank $\mathrm{P}$ value were calculated

\section{The Relationship Between CBX7 and Immune Microenvironment by TIMER}

TIMER(https://cistrome.shinyapps.io/timer/) ${ }^{16}$ is a web resource for systematical evaluations of the clinical impact of different immune cells among diverse cancer types. The level of abundance regarding six immune cell types: B cell, CD4 T cell, CD8 T cell, neutrophil, macrophage and dendritic cell in the tumor microenvironment can be estimated. Gene markers of tumor-infiltrating immune cells included markers of CD8+T cells, T cells (general), B cells, monocytes, TAMs, M1macrophages, M2 macrophages, neutrophils, natural killer (NK)cells, dendritic cells (DCs), T-helper 1 (Th1) cells, T-helper 2(Th2) cells, follicular helper $\mathrm{T}$ (Tfh) cells, T-helper 17 (Th17) cells, Tregs, and exhausted T cells. These gene markers have been referred in prior studies. In current study, we used TIMER to explore the relationship between CBX7 and gene markers of immune infiltrating cells in LUAD. 


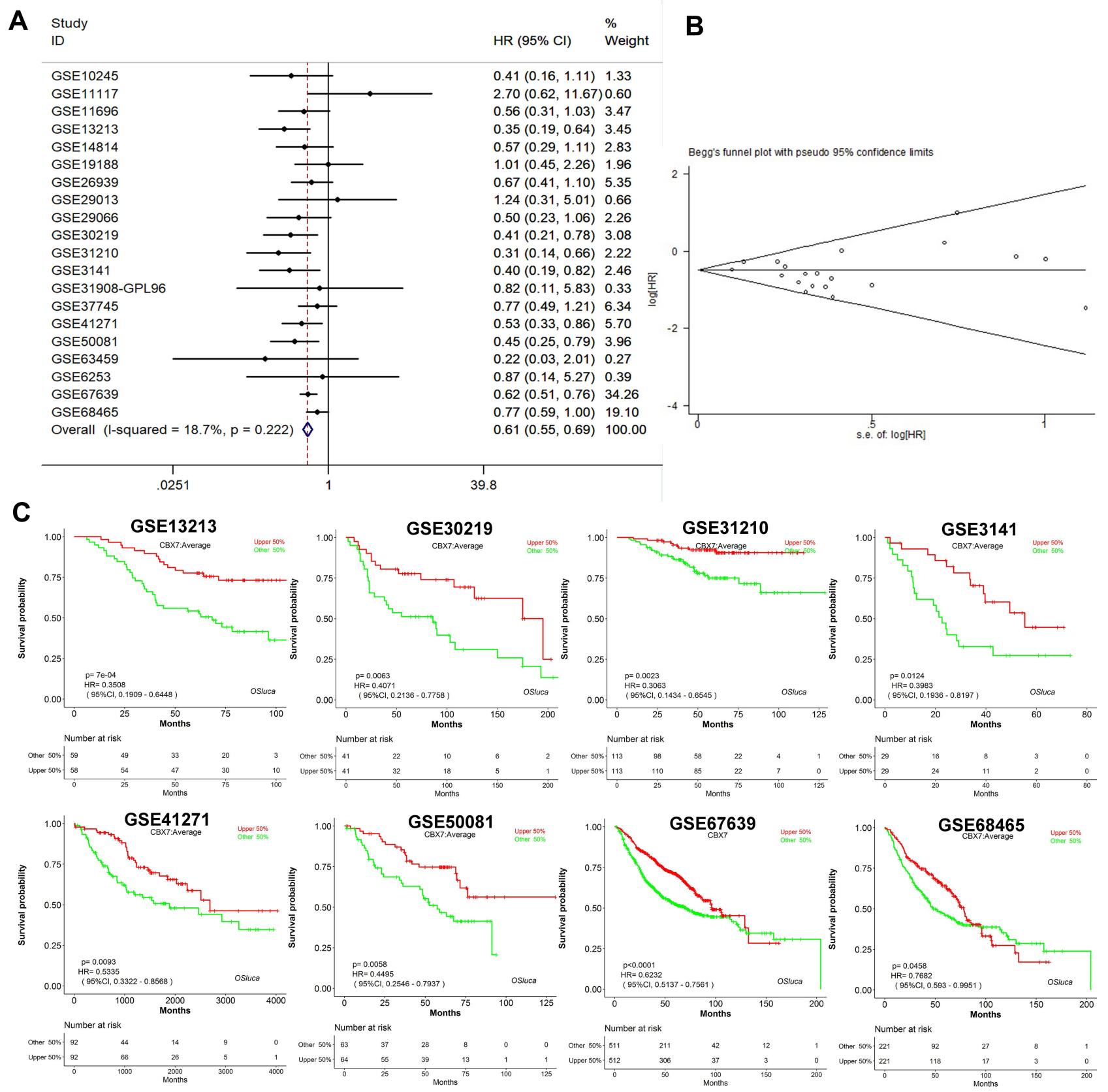

Figure 2 Meta-analyses of CBX7 expression in GEO dataset in OSluca online database. (A). Forrest plot of hazard ratio (HR) for the association of CBX7 expression with overall survival (OS) in LUAD. (B). The funnel plot of the meta-analysis of the impact of CBX7 expression on overall survival (OS) in LUAD. (C). Kaplan-Meier curves of OS for CBX7 expression in LUAD in GSEI3213, GSE30219, GSE31210, GSE3 I4I, GSE4I27I, GSE5008I, GSE67639 and GSE68465.

\section{Gene Set Enrichment Analysis (GSEA)}

GSEA was used to enrich the mRNAs predicted to be correlated with pathways. $\mathrm{P}<0.05$ was considered to indicate statistical significance, with reference to gene sets from the Molecular Signatures Database (MSigDB) of GO gene sets and KEGG gene sets, respectively. The number of permutations was set at 1000 . Enrichment results satisfying a numerical $\mathrm{P}$-value $<0.05$ and a false discovery rate (FDR) $<0.25$ were considered to be statistically significant.

\section{Immunohistochemistry (IHC) Staining}

95 LUAD tissues and corresponding adjacent normal lung tissues were collected from patients receiving surgery at the Department of Thoracic Surgery, the First Affiliated Hospital of Kunming Medical University from Jan 2015 
Table 3 Summarized Meta-Analysis Results of the Impact of CBX7 Expression on Overall Survival in Patients with LUAD

\begin{tabular}{|c|c|c|c|c|c|c|c|}
\hline \multirow[t]{2}{*}{ Analysis } & \multirow{2}{*}{$\begin{array}{l}\text { No. of } \\
\text { Studies } \\
\text { (Patients) }\end{array}$} & \multirow[t]{2}{*}{ HR (95\% CI) } & \multirow[t]{2}{*}{$\mathbf{P}$} & \multicolumn{2}{|c|}{ Heterogeneity } & \multicolumn{2}{|c|}{ Publication Bias } \\
\hline & & & & $I^{2}(\%)$ & Phet & Begg's p & Egger's $p$ \\
\hline Overall & $20(2864)$ & $0.612(0.546,0.685)$ & $<0.001$ & 18.7 & 0.222 & $0.87 I$ & 0.456 \\
\hline \multicolumn{8}{|l|}{ Gender } \\
\hline Male & $17(1419)$ & $0.68 I(0.585,0.794)$ & $<0.001$ & 14.8 & 0.28 & 0.387 & 0.31 \\
\hline Female & $18(14 \mid 2)$ & $0.6 \mid 4(0.5 \mid 6,0.730)$ & $<0.001$ & 0 & 0.723 & 0.88 & 0.643 \\
\hline \multicolumn{8}{|l|}{ Age } \\
\hline$<60$ & $17(1080)$ & $0.592(0.48 I, 0.729)$ & $<0.001$ & 14.8 & 0.281 & 0.484 & 0.235 \\
\hline$>60$ & $17(1693)$ & $0.627(0.545,0.721)$ & $<0.001$ & 3 & 0.42 & 0.484 & 0.504 \\
\hline \multicolumn{8}{|l|}{ Smoking } \\
\hline Yes & $8(830)$ & $0.628(0.5 \mid 6,0.765)$ & $<0.001$ & 0 & 0.509 & 0.536 & 0.509 \\
\hline No & $6(233)$ & $0.830(0.483, I .424)$ & 0.498 & 25.4 & 0.244 & 0.707 & 0.631 \\
\hline \multicolumn{8}{|l|}{ Stage } \\
\hline I & II(I4I4) & $0.569(0.470,0.689)$ & $<0.001$ & 0 & 0.832 & 0.213 & 0.167 \\
\hline ॥ & $\mathrm{II}(4 \mid 5)$ & $0.66 \mathrm{I}(0.504,0.866)$ & 0.003 & 0 & 0.94 & 1 & 0.647 \\
\hline III & $7(265)$ & $0.734(0.557,0.993)$ & 0.044 & 0 & 0.746 & 0.368 & 0.689 \\
\hline
\end{tabular}

Abbreviation: Phet, P for Heterogeneity.

and Dec 2016. Anti-CBX7 (1:100 dilution, Cat. 148761-AP, ProteinTech, Wuhan, China) were used for IHC staining. Antibody staining was visualized with DAB and hematoxylin counterstain (ZSGB-Bio). The percentage of positively stained cells was scored on a scale of 0 to 4 as follows: $0:<1 \%, 1: 1-25 \%, 2: 25-50 \%, 3: 50-75 \%$ and 4 : $>75 \%$. The staining intensity was scored from 0 to 3 as follows: 0: negative, 1: weak, 2: moderate, and 3: strong. The immunoreactivity score (IRS) for each case was generated by multiplying percentages of positive cells and staining intensities. Immuno-stained sections were photographed using a microscope (Olympus Corporation, Tokyo, Japan).

\section{Statistical Analysis}

Kaplan-Meier survival analysis using log rank test was used to calculate the median survival time (MST). Univariate and multivariate Cox proportional hazards models were used to calculate the HR and 95\% CI. Statistical analyses were performed using $\mathrm{R}$ software v3.5.0 (R Foundation for Statistical Computing, Vienna, Austria) and GraphPad Prism v7.00 (GraphPad Software Inc., USA). $\mathrm{P}<0.05$ was considered statistically significant. Receiver operating characteristic (ROC) analysis for death and recurrence detection was performed to identify the Youden index for CBX7 expression (the cutoff to separate patients) in Kaplan Meier curves of OS and RFS.

\section{Result \\ Expression Profile and Clinicopathological Characteristics of CBX7 in LUAD in Oncomine and TCGA Datasets}

Five datasets ${ }^{17-21}$ from Oncomine were used to evaluate the CBX7 expression in LUAD and normal lung tissue, all of which demonstrated $\mathrm{CBX} 7$ was downregulated in LUAD compared with normal lung tissues. Fold change (FC) of LUAD exceeded 2 in 4 studies (Table 1). Furthermore, data of 59 normal lung tissues and 526 LUAD tissues were obtained from TCGA dataset to evaluate the expression and clinicopathological characteristics of $\mathrm{CBX} 7$ in LUAD. Expression level of $\mathrm{CBX} 7$ was found to be significantly downregulated in LUAD compared with normal lung tissues $(\mathrm{P}<0.001)$ (Figure 1A).

In addition, the expression profile of $\mathrm{CBX} 7$ in pancancer was also investigated by Oncomine and GEPIA databases. We found $\mathrm{CBX} 7$ was not only downregulated in LUAD but also downregulated in most cancers (Supplementary Figure S1), indicated CBX7 may play a tumor suppressor gene in many cancers.

495 LUAD patients with full clinicopathological characteristics were further analyzed. Low CBX7 expression was found to be more common in male, age $<65$ years, as well as smokers. Low CBX7 expression seemed to be associated with increased tumor size, 
A

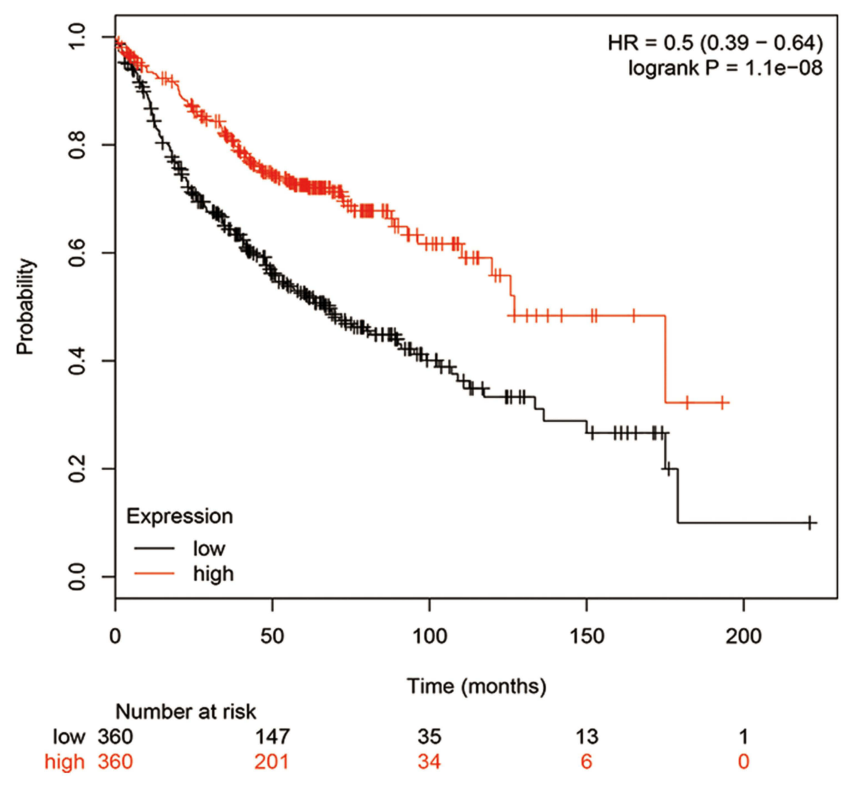

C

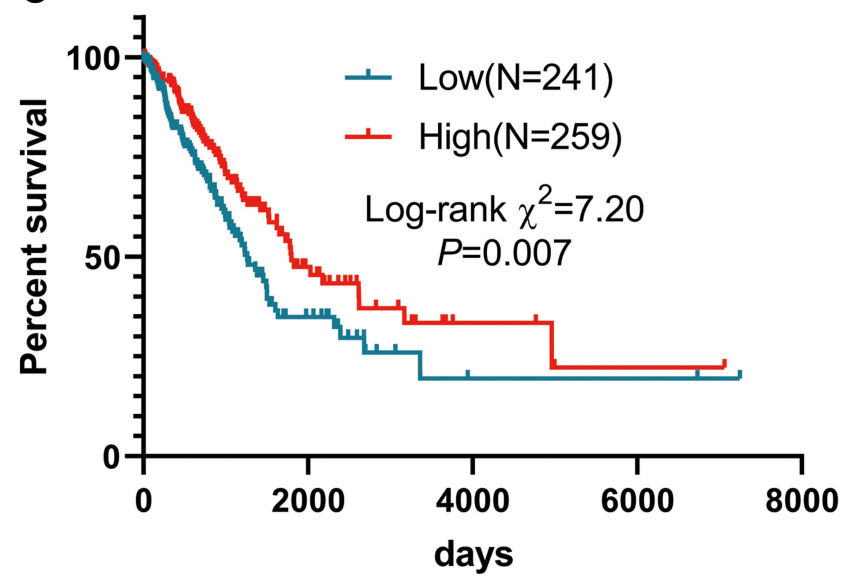

B

CBX7 (212914_at)
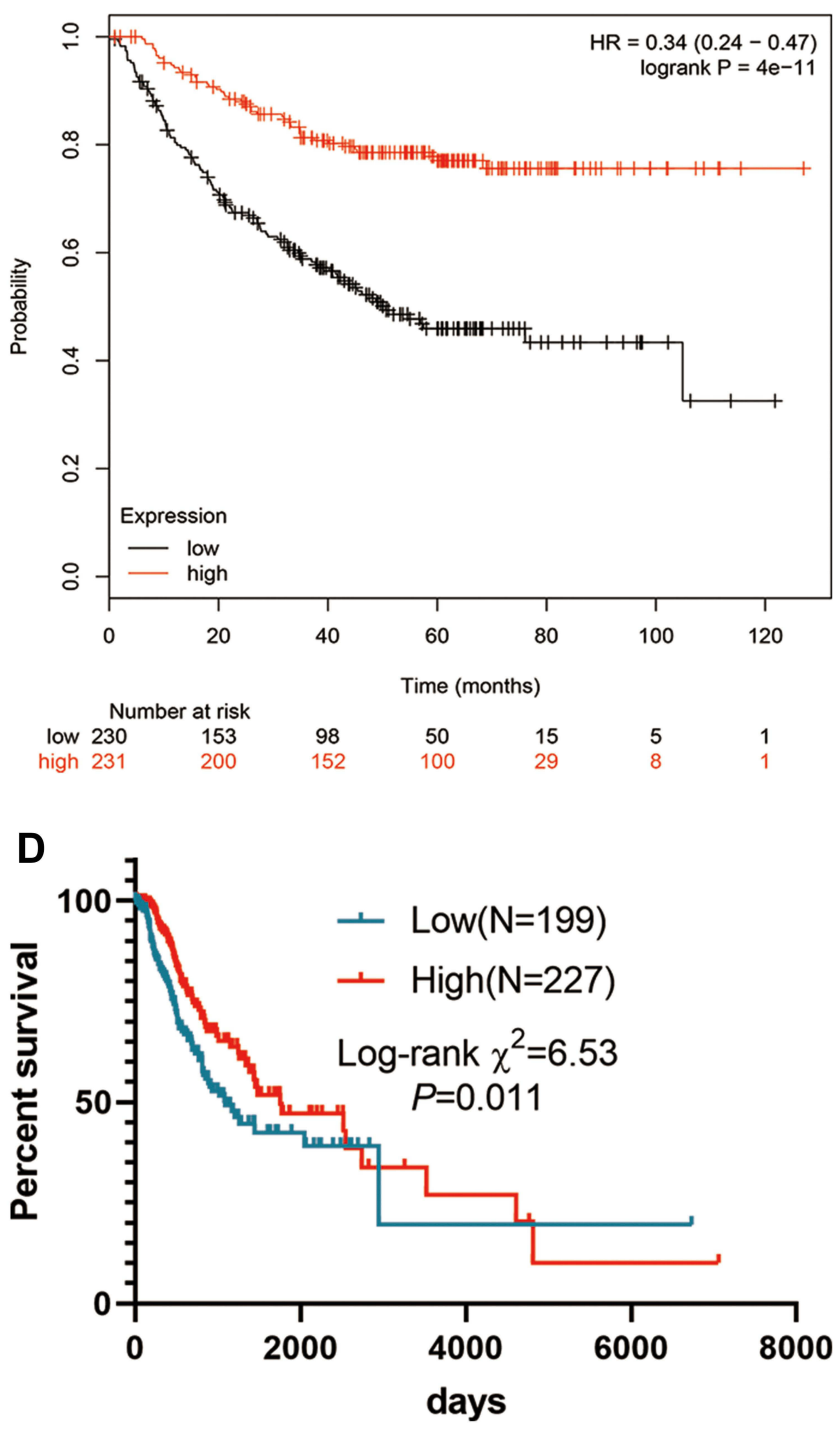

Figure 3 Kaplan-Meier curves of overall survival (OS) and recurrence-free survival (RFS) for CBX7 expression in LUAD. (A and B) Kaplan-Meier curves of OS (A) and RFS (B) in KM plotter. (C and D) Kaplan-Meier curves of OS (C) and RFS (D) in TCGA.

lymph node metastasis, distant metastasis and advanced TNM stage $(\mathrm{P}<0.05)$ (Figure 1). ROC analysis yielded the cutoff value for CBX7 as 2.0119. According to this cut-off value, 495 LUAD patients were divided into two groups, low CBX7 group $(\mathrm{n}=246)$ and high CBX7 group $(n=249)$. Similar results were obtained when continuous data was transformed into binary data. CBX7 expression level was found to be associated with age, sex, tumor size (T), lymph node metastasis (N), distant metastasis (M) and TNM stage. In addition, CBX7 expression was associated with recurrence and living status (Table 2).

\section{CBX7 Expression and OS in LUAD by} Meta-Analysis of OSluca Online Database A total of 20 GEO datasets including 2864 LUAD patients from OSluca were included for analysis. The pooled analysis suggested reduced $\mathrm{CBX} 7$ was associated with worse OS $(\mathrm{HR}=0.612,95 \% \mathrm{CI}=1.39-1.80, \mathrm{p}<0.001)$, with no heterogeneity between studies $\left(\mathrm{I}^{2}=18.7 \%, \quad \mathrm{P}=0.222\right)$ (Figure 2A and Table 3). The shape of the funnel plot was symmetrical for all comparisons suggesting no publication bias detected (Figure 2B). In subgroup analysis, most subgroups such as sex, age, smoking status and stage all suggested a significant association between low CBX7 
Table 4 Univariate and Multivariate Analysis of OS and RFS in LUAD Patients in TCGA Dataset

\begin{tabular}{|c|c|c|c|c|c|c|c|c|}
\hline & \multicolumn{4}{|c|}{ os } & \multicolumn{4}{|c|}{ RFS } \\
\hline & \multicolumn{2}{|c|}{ Univariate Analysis } & \multicolumn{2}{|c|}{ Multivariate Analysis } & \multicolumn{2}{|c|}{ Univariate Analysis } & \multicolumn{2}{|c|}{ Multivariate Analysis } \\
\hline & HR & $\mathbf{P}$ & HR & $\mathbf{P}$ & HR & $\mathbf{P}$ & HR & $\mathbf{P}$ \\
\hline Age (Continuous) & $1.008(0.993-1.024)$ & 0.282 & & & $1.010(0.993-1.026)$ & 0.257 & & \\
\hline $\begin{array}{l}\text { Gender } \\
\text { Male vs female }\end{array}$ & $1.04 I(0.777-1.395)$ & 0.787 & & & $0.892(0.645-1.233)$ & 0.49 & & \\
\hline $\begin{array}{l}\text { Smoking history } \\
\text { Yes vs no }\end{array}$ & $0.896(0.593-1.354)$ & 0.603 & & & $1.191(0.74 \mid-1.913)$ & 0.471 & & \\
\hline $\begin{array}{l}\text { T } \\
\text { T3-4 vs TI-2 }\end{array}$ & $2.367(1.615-3.468)$ & $<0.001$ & $1.735(1.133-2.658)$ & 0.011 & $2.023(1.244-3.287)$ & 0.004 & $1.721(1.032-2.870)$ & 0.048 \\
\hline $\begin{array}{l}\text { N } \\
\text { NI-3 vs No }\end{array}$ & $2.773(2.066-3.722)$ & $<0.001$ & $2.323(1.632-3.306)$ & $<0.001$ & $1.730(1.248-2.396)$ & 0.001 & $1.503(1.004-2.252)$ & 0.048 \\
\hline $\begin{array}{l}\text { M } \\
\text { MI vs MO }\end{array}$ & $2.315(1.382-3.877)$ & 0.001 & $1.480(0.806-2.718)$ & 0.206 & $1.388(0.648-2.974)$ & 0.399 & & \\
\hline $\begin{array}{l}\text { Pathologic Stage } \\
\text { III+IV vs I+II }\end{array}$ & $2.695(1.975-3.675)$ & $<0.001$ & $1.179(0.760-1.832)$ & 0.462 & $1.717(1.172-2.516)$ & 0.006 & $1.110(0.681-1.811)$ & 0.675 \\
\hline $\begin{array}{l}\text { CBX7 expression } \\
\text { (Continuous) }\end{array}$ & $0.673(0.533-0.848)$ & 0.001 & $0.749(0.584-0.959)$ & 0.022 & $0.693(0.545-0.882)$ & 0.003 & $0.712(0.553-0.917)$ & 0.009 \\
\hline
\end{tabular}

and poor OS with no heterogeneity (Table 3). Eight representative KM plots from OSluca online database (GSE13213, GSE30219, GSE31210, GSE3141, GSE41271, GSE50081, GSE67639 and GSE68465) are showed in Figure 2C.

\section{Prognostic Role of CBX7 in LUAD in KM Plotter and TCGA}

Firstly, KM plotter was used to assess the prognostic role of CBX7 in LUAD. Low CBX7 expression was found to be associated with poor $\mathrm{OS}(\mathrm{HR}=0.50,95 \% \mathrm{CI}=0.39-0.64$, $\left.\mathrm{P}=1.1 \times 10^{-8}\right) \quad$ (Figure $\left.3 \mathrm{~A}\right)$ and RFS $(\mathrm{HR}=0.34, \quad 95 \%$ $\mathrm{CI}=0.24-0.47, \mathrm{P}=4 \times 10^{-8}$ ) (Figure $3 \mathrm{~B}$ ). TCGA dataset analysis showed that patients with low $\mathrm{CBX} 7$ expression tend to have worse OS (Figure 3C) and RFS (Figure 3D). Moreover, univariate and multivariate Cox regression analyses were conducted. In univariate analysis, $\mathrm{T}, \mathrm{N}$, $\mathrm{M}$ pathologic stage and low $\mathrm{CBX} 7$ protein level were correlated with worse OS, while $\mathrm{T}, \mathrm{N}$ pathologic stage and low CBX7 protein level were correlated with worse RFS (Table 4). Further multivariate showed that only T, N and CBX7 were associated with poor OS and RFS (Table 4).

\section{CBX7 Expression Was Associated with Tumor-Infiltrating Immune Cells in LUAD} The relationship between $\mathrm{CBX} 7$ and immune infiltration in LUAD was evaluated by TIMER. CBX7 was found to be negatively correlated with tumor purity $(\mathrm{r}=-0.246, \mathrm{P}=3.2 \mathrm{e}-$ 8). There was a positive correlation between $\mathrm{CBX} 7$ expression and $\mathrm{B}$ cell $(\mathrm{r}=0.492, \mathrm{P}=7.52 \mathrm{e}-31), \mathrm{CD} 8+$ $\mathrm{T}$ cell $(\mathrm{r}=0.16, \quad \mathrm{P}=2.97 \mathrm{e}-4), \quad \mathrm{CD} 4+\mathrm{T}$ cell $(\mathrm{r}=0.49$, $\mathrm{P}=1.33 \mathrm{e}-33)$, macrophage $(\mathrm{r}=0.265, \mathrm{P}=3 \mathrm{e}-9)$, neutrophil

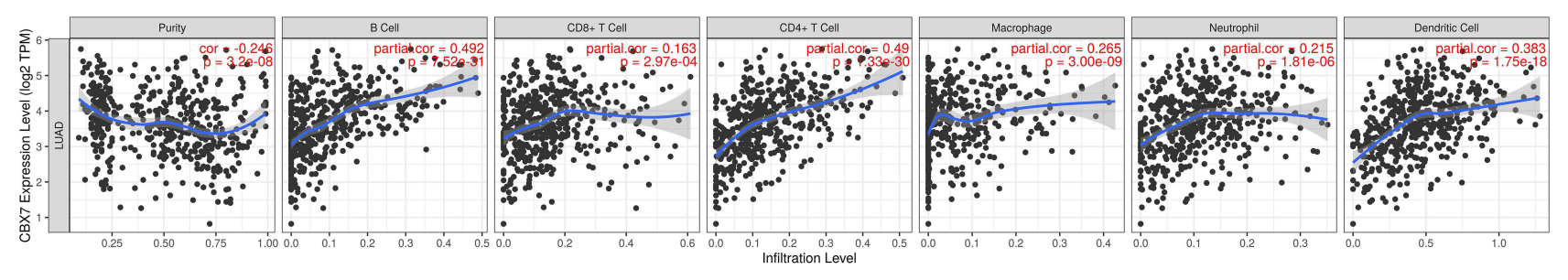

Figure 4 CBX7 expression level has significant negative correlations with infiltrating levels of B cell, CD4+ T cells, Macrophages, Neutrophils and DCs in LUAD. 
Table 5 The Relationship Between CBX7 and Relate Genes and Markers of Immune Cells in TIMER

\begin{tabular}{|c|c|c|c|c|c|}
\hline & & \multicolumn{2}{|l|}{ None } & \multicolumn{2}{|l|}{ Purity } \\
\hline & & Cor & $\mathbf{P}$ & Cor & $\mathbf{P}$ \\
\hline \multirow[t]{2}{*}{ CD8+ $T$ cell } & CD8A & 0.183 & 0.0000303 & 0.092 & 0.0403 \\
\hline & CD8B & 0.12 & 0.00632 & 0.047 & 0.299 \\
\hline \multirow[t]{3}{*}{ T cell (general) } & CD3D & 0.226 & $2.29 \mathrm{E}-7$ & 0.119 & 0.0083 \\
\hline & CD3E & 0.373 & I.78E-18 & 0.299 & I.22E-II \\
\hline & CD2 & 0.354 & $1.18 \mathrm{E}-16$ & 0.274 & $6.26 \mathrm{E}-10$ \\
\hline \multirow[t]{2}{*}{$B$ cell } & CDI9 & 0.413 & I.I5E-22 & 0.36 & $1.60 \mathrm{E}-16$ \\
\hline & CD79A & 0.352 & $1.68 \mathrm{E}-16$ & 0.292 & 3.66E-1I \\
\hline \multirow[t]{2}{*}{ Monocyte } & CD86 & 0.262 & I.58E-09 & 0.179 & 6. $19 \mathrm{E}-05$ \\
\hline & CDII5 (CSFIR) & 0.401 & $2.42 \mathrm{E}-2 \mathrm{I}$ & 0.341 & $7.14 \mathrm{E}-15$ \\
\hline \multirow[t]{3}{*}{ TAM } & CCL2 & 0.185 & $2.36 \mathrm{E}-05$ & 0.108 & I.04E-03 \\
\hline & CD68 & $0.28 I$ & $6.86 \mathrm{E}-\mathrm{II}$ & 0.22 & 8.47E-07 \\
\hline & ILIO & 0.284 & 4.9IE-II & 0.206 & $3.86 \mathrm{E}-06$ \\
\hline \multirow[t]{3}{*}{ MI Macrophage } & INOS (NOS2) & 0.126 & 4.15E-03 & 0.092 & 4. $12 \mathrm{E}-02$ \\
\hline & IRF5 & 0.378 & $6.47 \mathrm{E}-19$ & 0.325 & $1.34 \mathrm{E}-13$ \\
\hline & COX2(PTGS2) & -0.066 & 0.134 & -0.06 & 0.184 \\
\hline \multirow[t]{3}{*}{ M2 Macrophage } & CDI63 & 0.266 & $8.85 \mathrm{E}-10$ & 0.201 & $6.7 \mathrm{IE}-06$ \\
\hline & VSIG4 & 0.229 & I.53E-07 & 0.164 & 2.6IE-04 \\
\hline & MS4A4A & 0.261 & I.90E-09 & 0.187 & $2.98 \mathrm{E}-05$ \\
\hline \multirow[t]{3}{*}{ Neutrophils } & CD66b (CEACAM8) & 0.37 & $4.12 \mathrm{E}-18$ & 0.366 & 4.79E- 17 \\
\hline & CDIIb (ITGAM) & 0.437 & I.18E-25 & 0.381 & I.89E- 18 \\
\hline & CCR7 & 0.507 & $5.20 \mathrm{E}-35$ & 0.457 & 7.8IE-27 \\
\hline \multirow[t]{7}{*}{ Natural killer cell } & KIR2DLI & 0.039 & 0.377 & 0.002 & 0.967 \\
\hline & KIR2DL3 & 0.017 & 0.698 & -0.04 & 0.371 \\
\hline & KIR2DL4 & -0.14 & I.4IE-03 & -0.208 & 3.10E-06 \\
\hline & KIR3DLI & 0.048 & 0.279 & -0.006 & 0.889 \\
\hline & KIR3DL2 & 0.046 & 0.3 & -0.015 & 0.748 \\
\hline & KIR3DL3 & 0.088 & 4.59E-02 & -0.11 & I.47E-02 \\
\hline & KIR2DS4 & 0.059 & 0.181 & 0 & 0.998 \\
\hline \multirow[t]{7}{*}{ Dendritic cell } & HLA-DPBI & 0.547 & I.47E-4| & 0.509 & $8.03 E-34$ \\
\hline & HLA-DQBI & $0.44 I$ & $6.08 \mathrm{E}-26$ & 0.393 & $1.29 \mathrm{E}-19$ \\
\hline & HLA-DRA & 0.434 & $5.16 \mathrm{E}-25$ & 0.381 & $1.60 \mathrm{E}-18$ \\
\hline & HLA-DPAI & 0.507 & 6.IIE-35 & 0.465 & $7.76 \mathrm{E}-28$ \\
\hline & BDCA-I(CDIC) & 0.513 & $5.91 \mathrm{E}-36$ & 0.472 & $9.16 \mathrm{E}-29$ \\
\hline & BDCA-4(NRPI) & 0.117 & 8.09E-03 & 0.089 & 4.77E-02 \\
\hline & CDIIc(ITGAX) & 0.443 & 3.37E-26 & 0.391 & $1.87 \mathrm{E}-19$ \\
\hline \multirow[t]{5}{*}{ Thl } & T-bet(TBX2I) & 0.359 & $4.34 \mathrm{E}-17$ & 0.292 & 3.76E-II \\
\hline & STAT4 & 0.33 & I.57E-14 & 0.258 & $6.24 \mathrm{E}-09$ \\
\hline & STATI & 0.0042 & 0.341 & -0.034 & 0.447 \\
\hline & IFN- $\gamma($ IFNG) & -0.006 & 0.889 & -0.096 & $3.36 \mathrm{E}-02$ \\
\hline & TNF- $\alpha(T N F)$ & 0.314 & $3.17 \mathrm{E}-13$ & 0.24 & $6.64 \mathrm{E}-08$ \\
\hline \multirow[t]{4}{*}{ Th2 } & GATA3 & 0.324 & $4.53 \mathrm{E}-\mathrm{I} 4$ & 0.258 & $6.06 \mathrm{E}-09$ \\
\hline & STAT6 & 0.394 & I.36E-20 & 0.427 & 2.57E-23 \\
\hline & STAT5A & 0.544 & $5.22 \mathrm{E}-4 \mathrm{I}$ & 0.5 & $1.75 E-32$ \\
\hline & ILI3 & 0.158 & 3.08E-04 & 0.119 & $8.28 \mathrm{E}-03$ \\
\hline
\end{tabular}

(Continued) 
Table 5 (Continued).

\begin{tabular}{|c|c|c|c|c|c|}
\hline & & \multicolumn{2}{|l|}{ None } & \multicolumn{2}{|l|}{ Purity } \\
\hline & & Cor & $\mathbf{P}$ & Cor & $\mathbf{P}$ \\
\hline \multirow[t]{2}{*}{ Tfh } & BCL6 & 0.308 & $8.63 \mathrm{E}-13$ & 0.315 & $7.01 E-13$ \\
\hline & IL2। & 0.077 & $8.23 \mathrm{E}-02$ & 0.021 & 0.64 \\
\hline \multirow[t]{2}{*}{ ThI7 } & STAT3 & 0.268 & $6.77 \mathrm{E}-10$ & 0.284 & $1.28 \mathrm{E}-10$ \\
\hline & ILI7A & -0.013 & 0.761 & -0.068 & 0.133 \\
\hline \multirow[t]{4}{*}{ Treg } & FOXP3 & 0.323 & $6.13 \mathrm{E}-14$ & 0.251 & $1.55 \mathrm{E}-08$ \\
\hline & CCR8 & 0.319 & $1.15 \mathrm{E}-13$ & 0.246 & 3.IIE-08 \\
\hline & STAT5B & 0.479 & $5.75 E-31$ & 0.473 & 8.06E-29 \\
\hline & TGF $\beta$ (TGFBI) & 0.414 & $9.10 \mathrm{E}-23$ & 0.37 & 2. IIE-I7 \\
\hline \multirow[t]{5}{*}{$\mathrm{T}$ cell exhaustion } & PD-I (PDCDI) & 0.218 & $5.56 \mathrm{E}-07$ & 0.127 & $4.66 \mathrm{E}-03$ \\
\hline & CTLA4 & 0.27 & $4.4 \mathrm{IE}-10$ & 0.178 & 7.02E-05 \\
\hline & LAG3 & 0.117 & 7.95E-03 & 0.035 & 0.441 \\
\hline & TIM-3 (HAVCR2) & 0.236 & $6.00 \mathrm{E}-08$ & 0.148 & $9.75 \mathrm{E}-04$ \\
\hline & GZMB & -0.12 & $6.53 \mathrm{E}-03$ & -0.237 & $9.54 \mathrm{E}-08$ \\
\hline
\end{tabular}

$(\mathrm{r}=0.215, \quad \mathrm{P}=1.81 \mathrm{e}-6)$, and dendritic cell $\quad(\mathrm{r}=0.383$, $\mathrm{P}=1.75 \mathrm{e}-18$ ) (Figure 4 ). In addition, various immune marker genes corresponding to different tumor-infiltrating immune cells (CD8+ $\mathrm{T}$ cells, $\mathrm{T}$ cells (general), B cells, monocytes, TAMs, M1 and M2 macrophages, neutrophils, NK cells, Th1, Th2, Tfh, Th17, Treg and T cell exhaustion) were also investigated by TIMER and CBX7 were found to be associated with most of these marker genes. Generally, CBX7 expression were positively correlated with the majority of gene markers of different functional T cells (CD8+ T, Th1, Th2, Tfh, Th17, Treg and T cell exhaustion), B cells, dendritic cells, neutrophils and monocyte after purifying adjustments. $\mathrm{CBX} 7$ was related to tumor-associated macrophage (TAM, M1 and M2

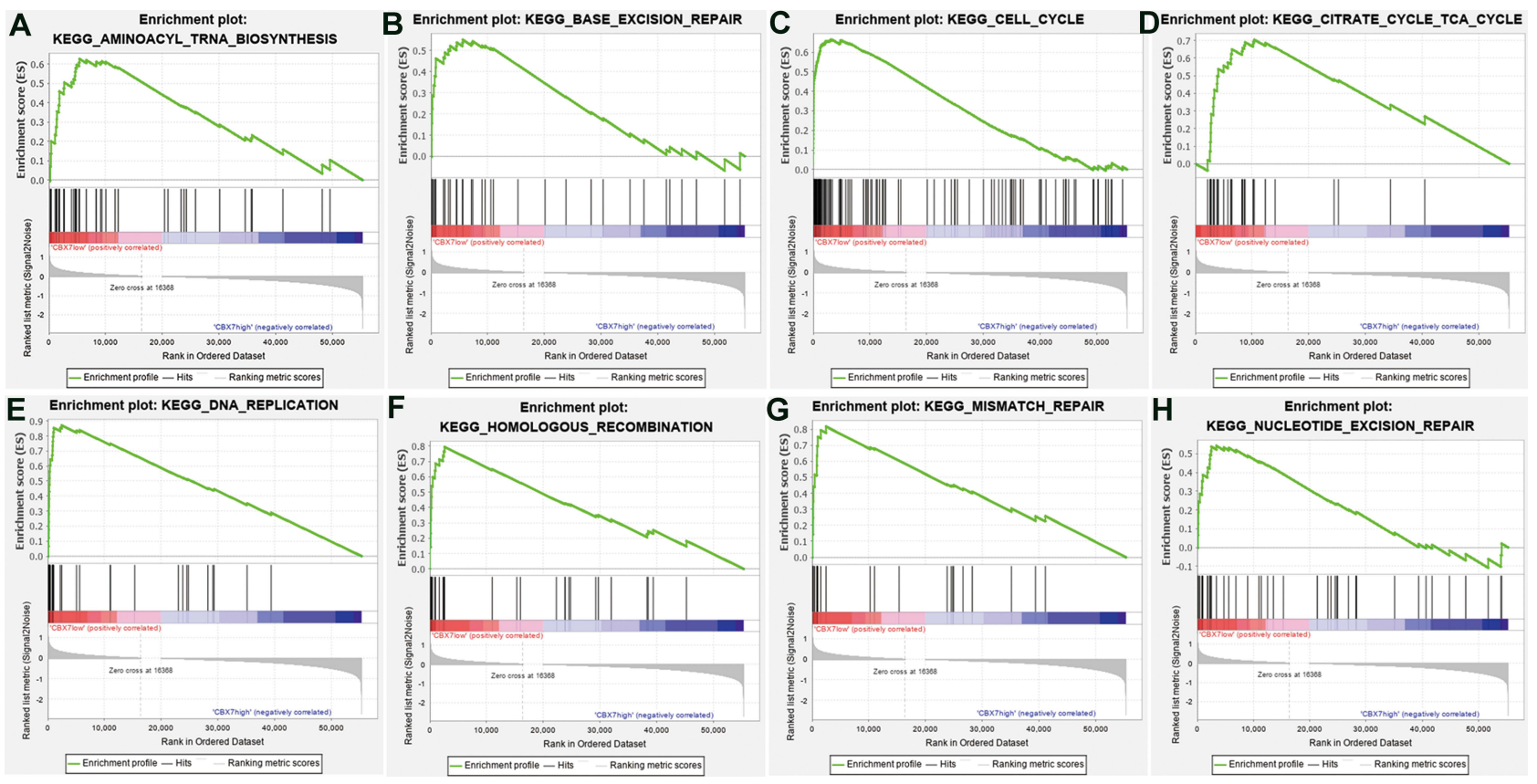

Figure 5 Gene Set Enrichment Analysis (GESA) results for tumors with low CBX7 expression in LUAD. 
Table 6 Gene Set Enrichment Analysis Result of CBX7 Low Expression Group

\begin{tabular}{|c|c|c|c|c|c|c|}
\hline & GS Details & Size & ES & NES & NOM P-val & FDR q-val \\
\hline I & KEGG_PYRIMIDINE_METABOLISM & 98 & 0.5940191 & 2.08511 & 0 & 0.022382332 \\
\hline 2 & KEGG_CITRATE_CYCLE_TCA_CYCLE & 31 & $0.70487 \mid 3$ & 2.0597153 & 0.010351967 & 0.0156989 \\
\hline 3 & KEGG_MISMATCH_REPAIR & 23 & 0.8160027 & 2.0580044 & 0 & 0.010465934 \\
\hline 4 & KEGG_PROTEASOME & 46 & 0.8175272 & $2.05587 I$ & 0 & 0.008497239 \\
\hline 5 & KEGG_HOMOLOGOUS_RECOMBINATION & 28 & 0.79443586 & 2.0513682 & 0 & 0.007408555 \\
\hline 6 & KEGG_DNA_REPLICATION & 36 & 0.87027 & 2.0502183 & 0 & 0.006319825 \\
\hline 7 & KEGG_CELL_CYCLE & 124 & 0.66358805 & 2.0225832 & 0 & 0.007860474 \\
\hline 8 & KEGG_PROTEIN_EXPORT & 24 & 0.6990288 & 2.0219886 & 0 & 0.006925017 \\
\hline 9 & KEGG_AMINOACYL_TRNA_BIOSYNTHESIS & $4 I$ & 0.62645996 & 1.9365052 & 0.01778656 & 0.01809742 \\
\hline 10 & KEGG_OXIDATIVE_PHOSPHORYLATION & $|3|$ & 0.65311223 & 1.8973855 & 0.018108651 & 0.026013676 \\
\hline 11 & KEGG_PARKINSONS_DISEASE & 127 & 0.6037786 & 1.8604995 & 0.024048096 & 0.032946516 \\
\hline 12 & KEGG_RNA_POLYMERASE & 29 & 0.59177357 & $1.8334 \mid 13$ & 0.017509727 & 0.038483705 \\
\hline 13 & KEGG_OOCYTE_MEIOSIS & 112 & 0.45340592 & $|.809953|$ & 0.013565891 & 0.043382503 \\
\hline 14 & KEGG_ALZHEIMERS_DISEASE & 165 & 0.4686439 & 1.8079304 & 0.016359918 & 0.041234728 \\
\hline 15 & KEGG_HUNTINGTONS_DISEASE & 180 & 0.46163434 & 1.7813784 & 0.026262626 & 0.04682553 \\
\hline 16 & KEGG_NUCLEOTIDE_EXCISION_REPAIR & 44 & 0.54154724 & 1.7526313 & 0.038387716 & 0.053722583 \\
\hline 17 & KEGG_RNA_DEGRADATION & 59 & 0.47982225 & 1.7003796 & $0.035928 \mid 45$ & 0.07108344 \\
\hline 18 & KEGG_ONE_CARBON_POOL_BY_FOLATE & 17 & 0.61857325 & 1.6639311 & 0.02745098 & 0.08360174 \\
\hline 19 & KEGG_BASE_EXCISION_REPAIR & 35 & 0.5514795 & 1.6436532 & $0.04357 \mid 43$ & 0.08530487 \\
\hline 20 & KEGG_RIBOFLAVIN_METABOLISM & 16 & 0.55264455 & 1.634612 & 0.022222223 & 0.08583631 \\
\hline 21 & KEGG_RIBOSOME & 87 & 0.7805994 & 1.60399 & 0.035196688 & 0.097854406 \\
\hline 22 & KEGG_GLYOXYLATE_AND_DICARBOXYLATE_METABOLISM & 16 & $0.547 \mid 389$ & 1.5795733 & 0.044624746 & 0.1067692 \\
\hline 23 & KEGG_PURINE_METABOLISM & 158 & 0.37173504 & I.5757207 & 0.0327553 & 0.104687735 \\
\hline 24 & KEGG_P53_SIGNALING_PATHWAY & 68 & 0.4271343 & 1.501431 & 0.043032788 & 0.13903996 \\
\hline
\end{tabular}

macrophage) infiltration (Table 5), which suggested that CBX7 play critical roles in immune microenvironment in LUAD.

\section{GSEA Result of CBX7}

Potential mechanism of CBX7 was explored and analyzed by GSEA. Pathways that were observed to be significantly enriched between $\mathrm{CBX} 7$ high $10 \%$ group and low $10 \%$ groups were analyzed. The result suggested that $\mathrm{CBX} 7$ may participate in Aminoacyl tRNA biosynthesis, base excision repair, cell cycle, citrate cycle TCA cycle, DNA replication, homologous recombination, mismatch repair, and nucleotide excision repair pathways (Figure 5 and Table 6).

\section{IHC Validation of CBX7 in LUAD}

95 paraffin-embedded LUAD and corresponding adjacent normal lung tissues were included for analysis. The IHC scores of paired LUAD tissues and their adjacent normal tissues showed that protein level of $\mathrm{CBX} 7$ is down-regulated in 95 LUAD patients $(\mathrm{P}<0.001)$ (Figure 6 and Figure 7A). Low CBX7 protein level was found to be correlated with differentiation degree, tumor size and lymph node metastasis (N). No significant association was found in region, sex, smoking status and TNM stage (Figure 7). The cutoff value yielded by ROC analysis for CBX7 IRS was 7.5, according to which patients were divided into low $\mathrm{CBX} 7(\mathrm{n}=46)$ and high CBX7 groups ( $\mathrm{n}=49)$. CBX7 expression was associated with living status, no significant association was found in age, region, sex, smoking history, differentiation degree, $\mathrm{T}$, N, M, TNM stage and recurrence status (Table 7). The prognostic role of $\mathrm{CBX} 7$ in LUAD was evaluated by univariate and multivariate Cox regression analyses. In univariate analysis, T, N, M, pathologic stage and low $\mathrm{CBX} 7$ protein level were correlated with the worst OS, while differentiation degree, N, M, pathologic stage and low $\mathrm{CBX} 7$ protein level were correlated with unfavorable RFS (Table 8 and Figure 8). In multivariate analysis, only pathologic $\mathrm{M}$ and $\mathrm{CBX} 7$ protein expression were associated with poor OS and RFS (Table 8).

\section{Discussion}

Our current study comprehensively investigated the prognostic role of $\mathrm{CBX} 7$ in LUAD. Through online database and our individual dataset, we demonstrated that $\mathrm{CBX} 7$ was down-regulated in LUAD tissues and might serve as an independent prognostic biomarker in LUAD. 
A

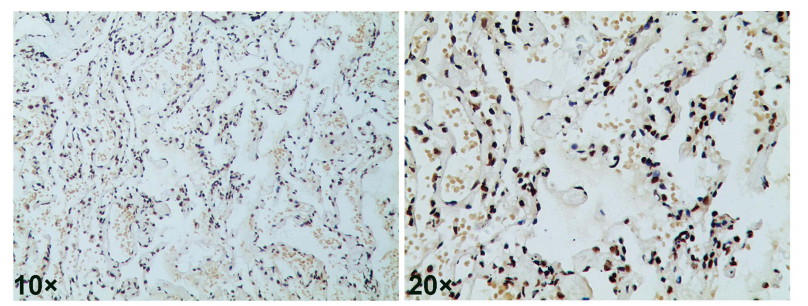

Adjacent normal lung tissue IRS12

C

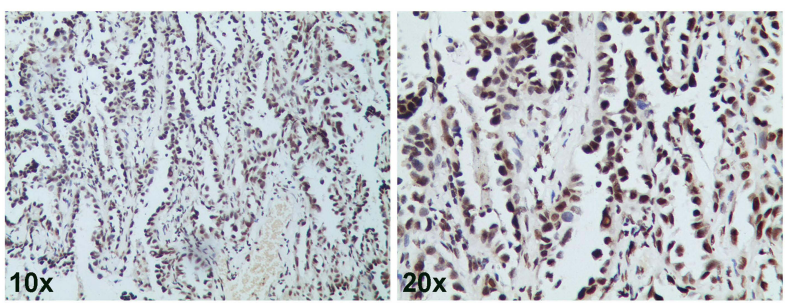

AD tissue IRS 12

E

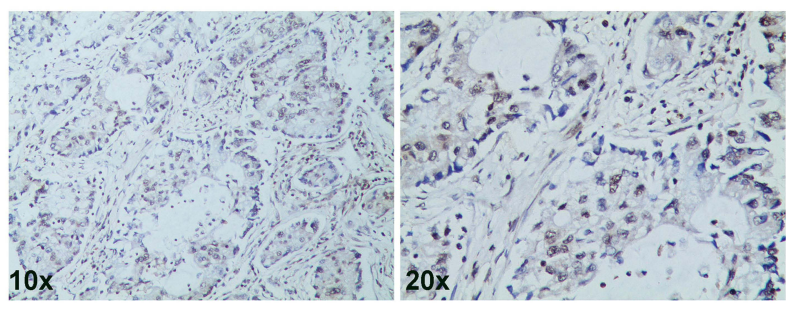

AD tissue IRS 2

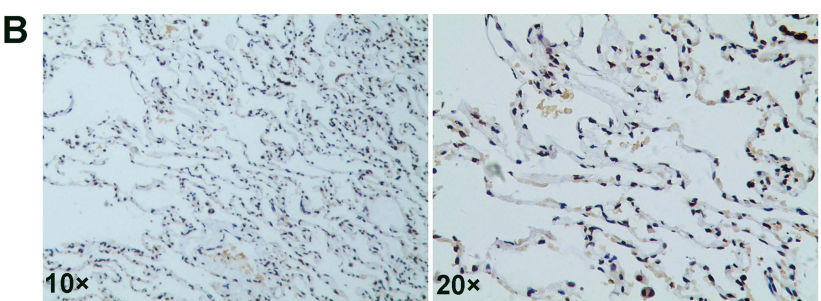

Adjacent Normal lung tissue IRS 8

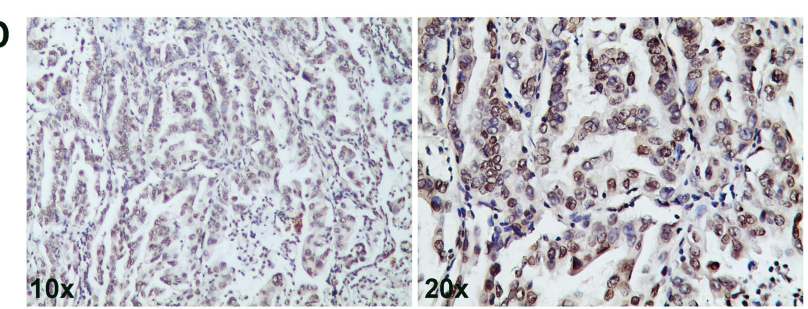

AD tissue IRS 8

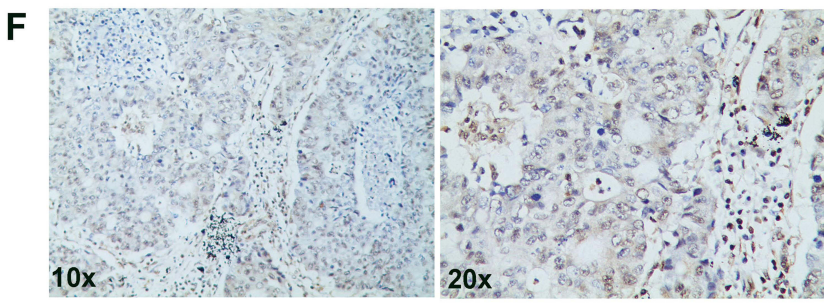

AD tissue IRS 1

Figure 6 Immunostaining of CBX7 in normal lung tissues and LUAD.
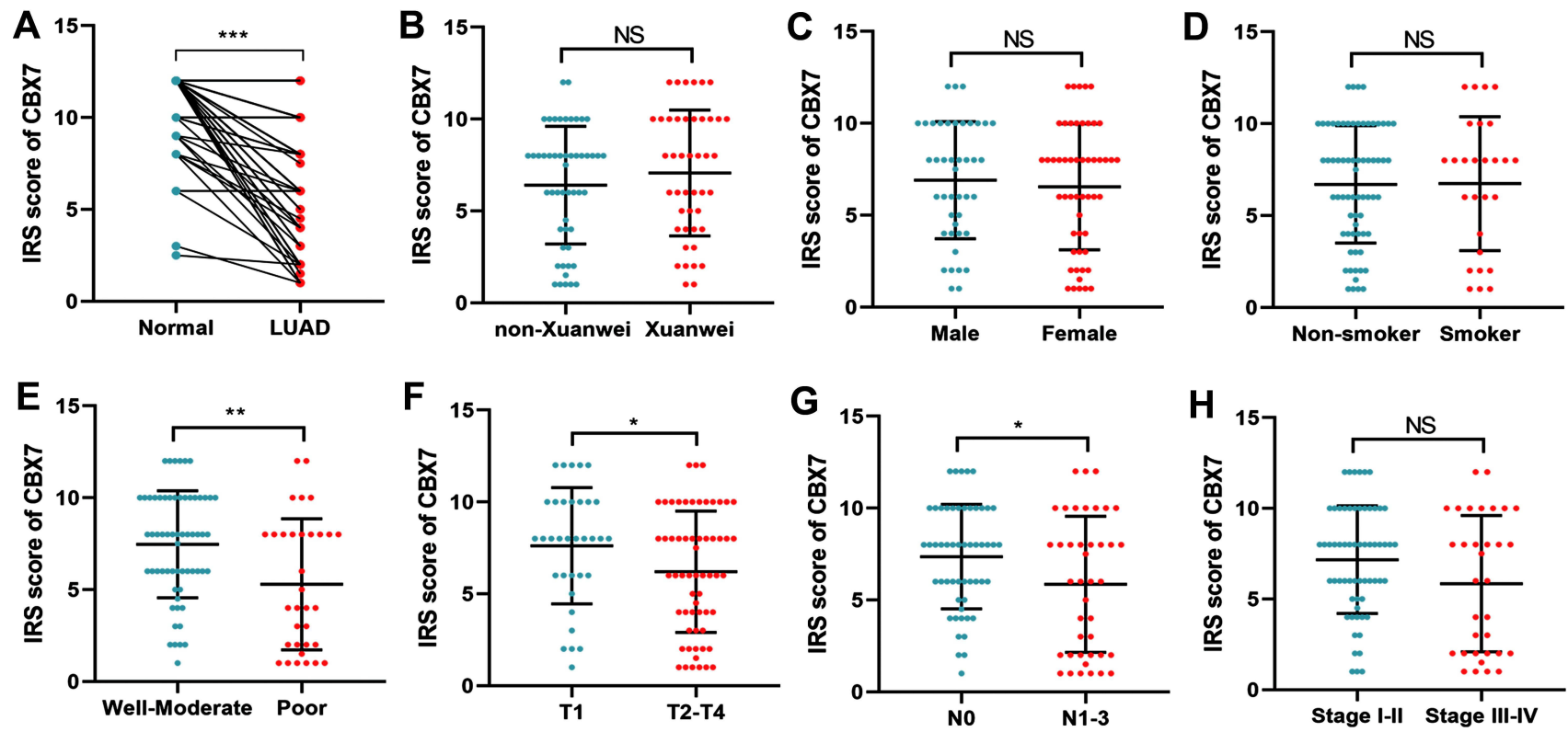

Figure 7 The expression level of CBX7 and its relationship with clinicopathological characteristics in 95 patients with LUAD. (A) CBX7 was downregulated in LUAD when compared with normal tissues. (B-H) The relationship between CBX7 expression and region (B), sex (C), smoking status (D), differentiation (E), T (F), N (G) and stage (H). ${ }^{*}, * *$ and $* * *$ represented $\mathrm{P}<0.05,0.01$ and 0.001 respectively. NS represented not significance. 
Table 7 The Relationship Between CBX7 and the Clinicopathological Characteristics in 95 patients with LUAD

\begin{tabular}{|c|c|c|c|}
\hline Parameters & Low $(N=46)$ & High $(N=49)$ & $\mathbf{P}$ \\
\hline Age & $55.3 \pm 11.1$ & $54.5 \pm 9.1$ & 0.001 \\
\hline Region & & & 0.9 \\
\hline Xuanwei & $21(45.7 \%)$ & $23(46.9 \%)$ & \\
\hline No-Xuanwei & $25(54.3 \%)$ & $26(53.1 \%)$ & \\
\hline Sex & & & \\
\hline Female & $21(45.7 \%)$ & $22(44.9 \%)$ & $0.94 I$ \\
\hline Male & $25(54.3 \%)$ & $27(55.1 \%)$ & \\
\hline Smoking History & & & \\
\hline Yes & $12(26.1 \%)$ & $15(30.6 \%)$ & 0.625 \\
\hline No & $34(73.9 \%)$ & $34(69.4 \%)$ & \\
\hline Differation & & & 0.193 \\
\hline Well+moderate & $27(58.7 \%)$ & $35(71.4 \%)$ & \\
\hline Poor & $19(41.3 \%)$ & $14(28.6 \%)$ & \\
\hline $\mathrm{T}$ & & & 0.056 \\
\hline TI & $12(26.1 \%)$ & $22(44.9 \%)$ & \\
\hline T2-T4 & $34(73.9 \%)$ & $27(55.1 \%)$ & \\
\hline $\mathrm{N}$ & & & 0.192 \\
\hline No & $23(50.0 \%)$ & $31(63.3 \%)$ & \\
\hline $\mathrm{NI}-3$ & $23(50.0 \%)$ & $18(36.7 \%)$ & \\
\hline$M$ & & & 0.25 \\
\hline Mo & $45(97.8 \%)$ & $44(89.8 \%)$ & \\
\hline MI & $\mathrm{I}(2.2 \%)$ & $5(10.2 \%)$ & \\
\hline Pathologic Stage & & & 0.399 \\
\hline I-II & $28(60.9 \%)$ & $34(69.4 \%)$ & \\
\hline III-IV & $18(39.1 \%)$ & $15(30.6 \%)$ & \\
\hline Recurrence status & & & 0.192 \\
\hline Living & $12(26.1 \%)$ & $20(40.8 \%)$ & \\
\hline Dead & $34(79.3 \%)$ & $29(59.2 \%)$ & \\
\hline Living Status & & & 0.032 \\
\hline Living & II (23.9\%) & $22(44.9 \%)$ & \\
\hline Dead & $35(76.1 \%)$ & $27(55.1 \%)$ & \\
\hline
\end{tabular}

LUAD and LUSC were the major types for NSCLC. With increasing prevalence and its dominant histology, LUAD has been selected as the focus of this study. Another important reason stands as to avoid bias that could possibly arise due to different biological processes and molecular profiles between LUAD and LUSC. Further studies are necessary to investigate the roles of $\mathrm{CBX} 7$ in LUSC and other subtypes in order to confirm whether CBX7 could serve as a potential biomarker in lung cancer treatment. As previous studies reported that $\mathrm{CBX7}$ was downregulated in many cancers including hepatocellular carcinoma, glioma, pancreatic cancer, colon, urothelial and thyroid cancer, such characteristic was also associated with poor prognosis in hepatocellular, glioma, pancreatic cancer and colon cancer. As far as human lung carcinoma is concerned, no detectable level of CBX7 has been detected in the samples mentioned, whereas $\mathrm{CBX} 7$ is abundantly expressed in normal lung tissue. Interestingly, $\mathrm{LOH}$ was reported in $50 \%$ of informative cases. Additionally, the expression of CBX7 was lost in the "normal" area surrounding carcinomas (non-pathological at the morphological evaluation) in $50 \%$ of the analyzed cases. Intriguingly, these cases also involved $\mathrm{LOH}$, suggesting that the decreased expression of CBX7 could be involved in the transition of epithelial lung cells toward a fully transformed and malignant phenotype. In this study, the expression profile and the prognostic role of CBX7 in LUAD have been comprehensively investigated using both online database and private database. Experimental results suggested that low CBX7 was associated with aggressive phenotypes, such as stage and definition, which is consistent with TCGA and private database analysis. Hence, this study is presented with rounded and scientifically sound evidence.

Reduced CBX7 expression was associated with aggressive phenotypes and poor survival status among lung cancer and other cancers, suggesting that CBX7 serves as a novel tumor-suppressive gene. Pallante et al stated that the loss of CBX7 promoted cancer development in mice, and CBX7knockout (CBX7-KO) mice were more likely to develop liver and lung neoplasia compared with wide-type. Mouse embryonic fibroblasts (MEFs) derived from CBX7-KO mice was used to explore the effect of $\mathrm{CBX} 7$ regarding cellular proliferation and $\mathrm{CBX} 7+/-$ and $\mathrm{CBX} 7-/-$ MEFs were found to grow faster than $\mathrm{CBX} 7+/+\mathrm{MEF}$. In addition, the percentage of S-phase cell in CBX7-KO MEF was higher than wildtype MEF. ${ }^{22}$ Several studies have shed light on the mechanism of reduced $\mathrm{CBX} 7$ promoting cancer development. It has been reported that $\mathrm{CBX7}$ was able to sustain the expression of the E-cadherin gene (CDH1) through binding to its promoter, as well as counteracting with anti-transcriptional effect of histone deacetylase 2 (HDAC2) and Protein Arginine Methyltransferase 1(PRMT1). ${ }^{23,24}$ As a result, the reduced expression of $\mathrm{CBX} 7$ decreases E-cadherin expression, which then leads to the critical event epithelial-tomesenchymal transition (EMT) ${ }^{23,24} \mathrm{CBX} 7$ binds the E-box to inhibit TWIST-1 function, tumorigenicity, and metastatic potential. Deletion of CBX7 in sEOC can sufficiently reactivate TWIST-1-induced transcription, prompt mesenchymal transformation, and enhanced tumorigenicity in vivo. ${ }^{25}$ CBX7 interacts with HMGA1b, which binds to and regulates 
Table 8 Univariate and Multivariate Analysis of OS and RFS in 95 Patients with LUAD

\begin{tabular}{|c|c|c|c|c|c|c|c|c|}
\hline & \multicolumn{4}{|c|}{ OS } & \multicolumn{4}{|c|}{ RFS } \\
\hline & \multicolumn{2}{|c|}{ Univariate Analysis } & \multicolumn{2}{|c|}{ Multivariate Analysis } & \multicolumn{2}{|c|}{ Univariate Analysis } & \multicolumn{2}{|c|}{ Multivariate Analysis } \\
\hline & HR $(95 \% \mathrm{Cl})$ & $\mathbf{P}$ & HR (95\% Cl) & $\mathbf{P}$ & HR (95\% Cl) & $\mathbf{P}$ & HR (95\% CI) & $\mathbf{P}$ \\
\hline Age (Continuous) & $1.007(0.98 \mathrm{I}-1.033)$ & 0.621 & & & $1.010(0.985-1.035)$ & 0.452 & & \\
\hline \multicolumn{9}{|l|}{ Gender } \\
\hline Male vs female & $1.174(0.708-1.947)$ & 0.534 & & & $1.161(0.706-1.910)$ & 0.557 & & \\
\hline \multicolumn{9}{|l|}{ Region } \\
\hline Xuanwei vs Non-Xusanwei & $0.656(0.393-1.095)$ & 0.107 & & & $0.691(0.418-1.144)$ & 0.151 & & \\
\hline \multicolumn{9}{|l|}{ Smoking history } \\
\hline Yes vs no & $0.895(0.506-1.584)$ & 0.703 & & & $0.816(0.462-1.439)$ & 0.482 & & \\
\hline \multicolumn{9}{|l|}{ Differentiation } \\
\hline Poor vs well+moderate & $1.526(0.908-2.566)$ & 0.111 & & & $1.790(1.081-2.965)$ & 0.024 & $1.242(0.719-2.146)$ & 0.436 \\
\hline \multicolumn{9}{|l|}{$\mathrm{T}$} \\
\hline T2-4 vs TI & $1.822(1.039-3.197)$ & 0.036 & $1.278(0.700-2.333)$ & 0.425 & $1.720(0.994-2.977)$ & 0.053 & & \\
\hline \multicolumn{9}{|l|}{$N$} \\
\hline $\mathrm{NI}-3$ vs N0 & $2.840(1.691-4.796)$ & $<0.001$ & $1.276(0.508-3.208)$ & 0.604 & $2.54 I(I .533-4.209)$ & $<0.001$ & $1.346(0.554-3.27 I)$ & 0.511 \\
\hline \multicolumn{9}{|l|}{ M } \\
\hline MI vs M0 & $4.621(1.959-10.900)$ & $<0.001$ & $4.075(1.513-10.972)$ & 0.005 & $4.014(1.705-9.449)$ & 0.001 & $3.055(1.165-8.012)$ & 0.023 \\
\hline \multicolumn{9}{|l|}{ Pathologic Stage } \\
\hline III+IV vs I+II & $2.969(1.763-5.000)$ & $<0.001$ & $1.665(0.665-4.168)$ & 0.276 & $2.656(1.596-4.420)$ & $<0.001$ & $1.545(0.622-3.84 \mid)$ & 0.349 \\
\hline CBX7 expression (Continuous) & $0.869(0.802-0.942)$ & 0.001 & $0.877(0.805-0.955)$ & 0.003 & $0.892(0.824-0.965)$ & 0.004 & $0.902(0.829-0.982)$ & 0.018 \\
\hline
\end{tabular}

the acetylation of CCNE1 promoter. As a coding gene for cyclin E, CCNE1 regulates cell cycle that is crucial for G1/S transition. Another mechanism through which $\mathrm{CBX} 7$ involves with cancer progression is that $\mathrm{CBX} 7$ negatively modulates the expression of SPP1 gene that encodes the osteopontin protein, a chemokine promoting cell migration. ${ }^{23,26}$

This study also investigated the potential role of $\mathrm{CBX} 7$ in LUAD via GSEA analysis. Several cancer-related pathways were identified including aminoacyl tRNA biosynthesis, base excision repair, cell cycle, citrate cycle TCA cycle, DNA replication, homologous recombination, mismatch repair, and nucleotide excision repair pathways. All of these pathways have been proved to be closely related to cancer development, and some, for example, cell cycle pathways, have been reported and investigated by previous studies. ${ }^{22,23}$ How CBX7 participate in DNA damage repair pathways (base excision repair, DNA replication, homologous recombination, mismatch repair, and nucleotide excision repair pathways)? PcG proteins participate in DNA damage response (DDR). The majority of PcG proteins, including EZH2 and the CBXs, localize to regions of damaged chromatin in order to recruit DNA damage repair machinery. ${ }^{27,28}$ Inhibiting CBXs may enhance chemotherapeutic response by preventing DNA damage repair, allowing massive accumulation of DNA damage. ${ }^{29}$ However, the role of $\mathrm{CBX} 7$ in the process is still unknown. Further studies need to be conducted for verification.

Tumor microenvironments, especially tumor-infiltrating immune cells, play an important role in the prognosis of lung cancer. This study demonstrated that CBX7 affects and mediates immune infiltration level in LUAD. CBX7 expression was positively related to the level of B cells, CD8+ T cells, CD4+ T cells, macrophages, neutrophils and DCs infiltration. CBX7 expression was also positively related to most gene markers of $\mathrm{T}$ cells, B cells, tumor-associated macrophages (TAM), M1, M2, DC cell, and T helper cells (Th1, Th2, Tfh, and Th17). High level of these tumor-infiltrating immune was associated with better survival in lung cancer. CBX7 could regulate immune infiltration level by mediating gene expression in these cells. Also, in the pathway enriched analysis, we found that CBX may participate in DNA damage repair pathways (base excision repair, DNA replication, homologous recombination, mismatch repair, and nucleotide excision repair 

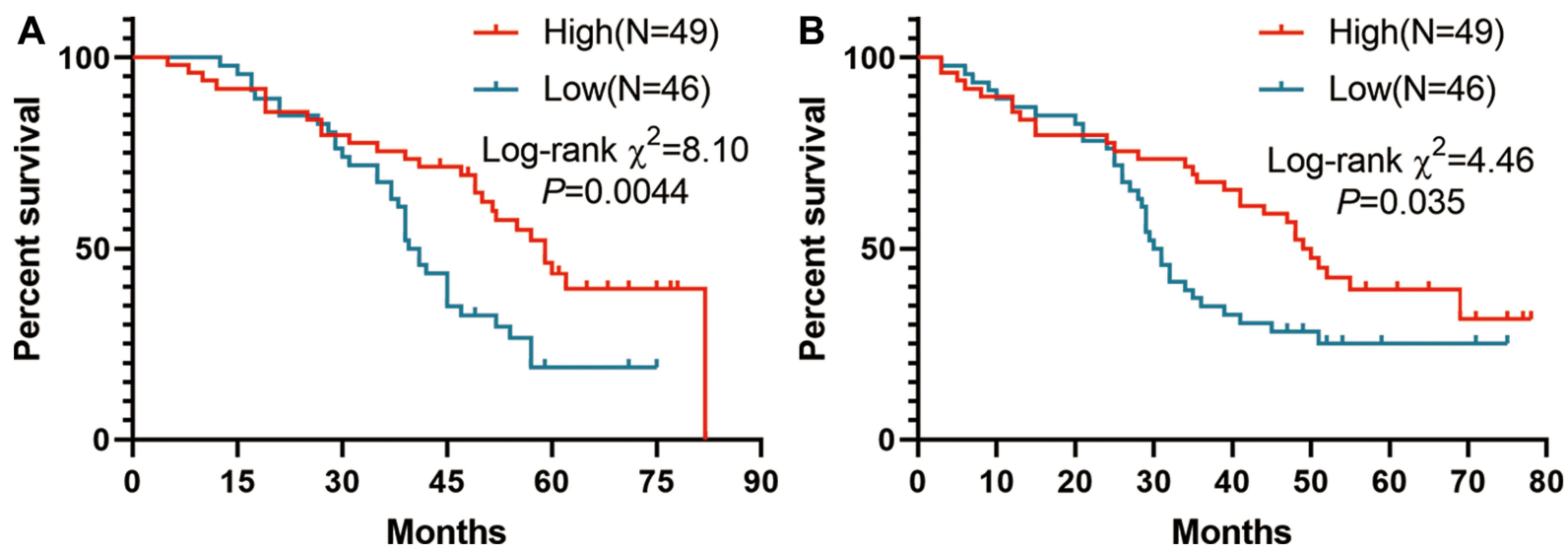

Figure 8 Kaplan-Meier curves of overall survival (OS)(A) and recurrence-free survival(RFS) (B) for CBX7 expression in 95 patients with LUAD.

pathways). The deficiency of DNA damage repair pathways may result in the high tumor mutation burden (TMB), which may be associated with immune infiltration. However, the precise role of CBX7 in tumor immune microenvironment and tumor progression still needs exploration through future studies.

To validate the potential role of $\mathrm{CBX} 7,95$ paraffinembedded LUAD and corresponding adjacent normal lung tissue samples were included for analysis. CBX7 was found to be downregulated in LUAD tissues and was associated with malignant phenotypes such as T/N/M. In addition, multiple cox analysis showed that $\mathrm{CBX} 7$ is an independent prognostic factor, indicating that $\mathrm{CBX} 7$ might serve as a useful prognostic biomarker in LUAD.

In conclusion, this study demonstrated that $\mathrm{CBX7}$ is downregulated in LUAD, which is associated with unappealing clinicopathological features and poor prognosis proved by both online datasets and our clinical samples. CBX7 stands as a crucial mediating factor regarding LUAD tumorigenesis due to its correlation with several cancer-related pathways and its regulating effects on immune infiltration level. Hence, CBX7 might serve as a promising biomarker and potential molecular target regarding clinical treatments of LUAD. Future large cohort and mechanism studies are necessary to validate and further reveal the molecular mechanisms and pathways of CBX7 in LUAD.

\section{Ethical Approval and Consent to Participate}

95 surgical LUAD samples used in this manuscript have been conducted in accordance with the principles of the 1964 Helsinki declaration and its later amendments or comparable ethical standards. Our study was also approved by the Ethics Committee of the First Affiliated Hospital of Kunming Medical University. All patients were obtained with informed consent.

\section{Author Contributions}

Yunping Zhao and Xiaobo Chen: conception and design of the work. Yanlong Yang and Zaoxiu Hu: conception, design of the work and have drafted the work. Linzhu Yang: the acquisition, analysis. Hongwen Sun and Qinghe Yu: substantively revised. Fang Yin, Yongmeng Sun, Xinming Zhu, Shen Li, Zaoxiu $\mathrm{Hu}$ and Lisha $\mathrm{Pu}$ collected data and analyzed. All authors contributed to data analysis, drafting or revising the article, have agreed on the journal to which the article will be submitted, gave final approval of the version to be published, and agree to be accountable for all aspects of the work.

\section{Funding}

This study was supported by Scientific Research Foundation of Yunnan Education Department (2019J1234 and 2019J1282), Yunnan Provincial Department of Science and Technology-Kunming Medical University Joint Foundation (202001AY070001-144 and 202101AY070001-172), Doctoral Research Foundation of the First Affiliated Hospital of Kunming Medical University (2018BS012) and Science and Technology Personnel Training Program of High-level Health Technicians in Yunnan Province (H-2017014). 


\section{Disclosure}

The authors have no conflicts of interest to declare.

\section{References}

1. Bray F, Ferlay J, Soerjomataram I, Siegel RL, Torre LA, Jemal A. Global cancer statistics 2018: GLOBOCAN estimates of incidence and mortality worldwide for 36 cancers in 185 countries. $C A$ Cancer J Clin. 2018;68(6):394-424. doi:10.3322/caac.21492

2. Herbst RS, Heymach JV, Lippman SM. Lung cancer. $N$ Engl J Med. 2008;359(13):1367-1380. doi:10.1056/NEJMra0802714

3. Zappa C, Mousa SA. Non-small cell lung cancer: current treatment and future advances. Transl Lung Cancer Res. 2016;5(3):288-300. doi:10.21037/tlcr.2016.06.07

4. Denisenko TV, Budkevich IN, Zhivotovsky B. Cell death-based treatment of lung adenocarcinoma. Cell Death Dis. 2018;9(2):117. doi:10.1038/s41419-017-0063-y

5. Pallante P, Forzati F, Federico A, Arra C, Fusco A. Polycomb protein family member $\mathrm{CBX} 7$ plays a critical role in cancer progression. $\mathrm{Am}$ $J$ Cancer Res. 2015;5(5):1594-1601.

6. Ma RG, Zhang Y, Sun TT, Cheng B. Epigenetic regulation by polycomb group complexes: focus on roles of CBX proteins. $J$ Zhejiang Univ Sci B. 2014;15(5):412-428. doi:10.1631/jzus.B1400077

7. Li R, Yan Q, Tian P, et al. CBX7 inhibits cell growth and motility and induces apoptosis in cervical cancer cells. Mol Ther Oncolytics. 2019;15:108-116. doi:10.1016/j.omto.2019.09.002

8. Ni S, Wang H, Zhu X, et al. CBX7 suppresses cell proliferation, migration, and invasion through the inhibition of PTEN/Akt signaling in pancreatic cancer. Oncotarget. 2017;8(5):8010-8021. doi:10.18632/oncotarget. 14037

9. Rhodes DR, Yu J, Shanker K, et al. ONCOMINE: a cancer microarray database and integrated data-mining platform. Neoplasia. 2004;6(1):1-6. doi:10.1016/S1476-5586(04)80047-2

10. Tang Z, Li C, Kang B, Gao G, Li C, Zhang Z. GEPIA: a web server for cancer and normal gene expression profiling and interactive analyses. Nucleic Acids Res. 2017;45(W1):W98-W102. doi:10.1093/nar/gkx247

11. Yan Z, Wang Q, Lu Z, et al. OSluca: an interactive web server to evaluate prognostic biomarkers for lung cancer. Front Genet. 2020;11:420. doi:10.3389/fgene.2020.00420

12. Mantel N, Haenszel W. Statistical aspects of the analysis of data from retrospective studies of disease. $J$ Natl Cancer Inst. 1959;22 (4):719-748.

13. DerSimonian R, Laird N. Meta-analysis in clinical trials. Control Clin Trials. 1986;7(3):177-188. doi:10.1016/0197-2456(86)90046-2

14. Begg CB, Mazumdar M. Operating characteristics of a rank correlation test for publication bias. Biometrics. 1994;50(4):1088-1101. doi: $10.2307 / 2533446$

15. Gyorffy B, Surowiak P, Budczies J, Lanczky A. Online survival analysis software to assess the prognostic value of biomarkers using transcriptomic data in non-small-cell lung cancer. PLoS One. 2013;8 (12):e82241. doi:10.1371/journal.pone.0082241
16. Li T, Fan J, Wang B, et al. TIMER: a web server for comprehensive analysis of tumor-infiltrating immune cells. Cancer Res. 2017;77(21): e108-e110. doi:10.1158/0008-5472.CAN-17-0307

17. Hou J, Aerts J, den Hamer B, et al. Gene expression-based classification of non-small cell lung carcinomas and survival prediction. PLoS One. 2010;5(4):e10312. doi:10.1371/journal.pone.0010312

18. Su LJ, Chang CW, Wu YC, et al. Selection of DDX5 as a novel internal control for Q-RT-PCR from microarray data using a block bootstrap re-sampling scheme. BMC Genomics. 2007;8:140. doi:10.1186/1471-2164-8-140

19. Selamat SA, Chung BS, Girard L, et al. Genome-scale analysis of DNA methylation in lung adenocarcinoma and integration with mRNA expression. Genome Res. 2012;22(7):1197-1211. doi:10.1101/gr.132662.111

20. Landi MT, Dracheva T, Rotunno M, et al. Gene expression signature of cigarette smoking and its role in lung adenocarcinoma development and survival. PLoS One. 2008;3(2):e1651. doi:10.1371/journal. pone. 0001651

21. Okayama H, Kohno T, Ishii $Y$, et al. Identification of genes upregulated in ALK-positive and EGFR/KRAS/ALK-negative lung adenocarcinomas. Cancer Res. 2012;72(1):100-111. doi:10.1158/ 0008-5472.CAN-11-1403

22. Forzati F, Federico A, Pallante P, et al. CBX7 is a tumor suppressor in mice and humans. J Clin Invest. 2012;122(2):612-623. doi:10.1172/ JCI58620

23. Federico A, Pallante P, Bianco M, et al. Chromobox protein homologue 7 protein, with decreased expression in human carcinomas, positively regulates E-cadherin expression by interacting with the histone deacetylase 2 protein. Cancer Res. 2009;69(17):7079-7087. doi:10.1158/0008-5472.CAN-09-1542

24. Federico A, Sepe R, Cozzolino F, et al. The complex CBX7-PRMT1 has a critical role in regulating E-cadherin gene expression and cell migration. Biochim Biophys Acta Gene Regul Mech. 2019;1862 (4):509-521. doi:10.1016/j.bbagrm.2019.02.006

25. Li J, Alvero AB, Nuti S, et al. CBX7 binds the E-box to inhibit TWIST-1 function and inhibit tumorigenicity and metastatic potential. Oncogene. 2020;39(20):3965-3979. doi:10.1038/s41388020-1269-5

26. Sepe R, Formisano U, Federico A, et al. CBX7 and HMGA1b proteins act in opposite way on the regulation of the SPP1 gene expression. Oncotarget. 2015;6(5):2680-2692. doi:10.18632/ oncotarget. 2777

27. Chou DM, Adamson B, Dephoure NE, et al. A chromatin localization screen reveals poly (ADP ribose)-regulated recruitment of the repressive polycomb and NuRD complexes to sites of DNA damage. Proc Natl Acad Sci U S A. 2010;107(43):18475-18480. doi:10.1073/ pnas. 1012946107

28. Gieni RS, Ismail IH, Campbell S, Hendzel MJ. Polycomb group proteins in the DNA damage response: a link between radiation resistance and "stemness". Cell Cycle. 2011;10(6):883-894. doi:10.4161/cc.10.6.14907

29. Connelly KE, Martin EC, Dykhuizen EC. CBX chromodomain inhibition enhances chemotherapy response in glioblastoma multiforme. Yale J Biol Med. 2016;89(4):431-440. 


\section{Publish your work in this journal}

OncoTargets and Therapy is an international, peer-reviewed, open access journal focusing on the pathological basis of all cancers, potential targets for therapy and treatment protocols employed to improve the management of cancer patients. The journal also focuses on the impact of management programs and new therapeutic

Submit your manuscript here: https://www.dovepress.com/oncotargets-and-therapy-journal agents and protocols on patient perspectives such as quality of life, adherence and satisfaction. The manuscript management system is completely online and includes a very quick and fair peer-review system, which is all easy to use. Visit http://www.dovepress.com/ testimonials.php to read real quotes from published authors. 